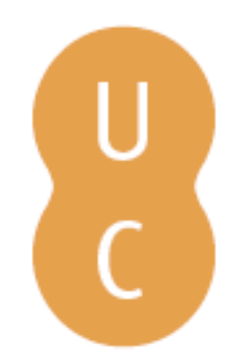

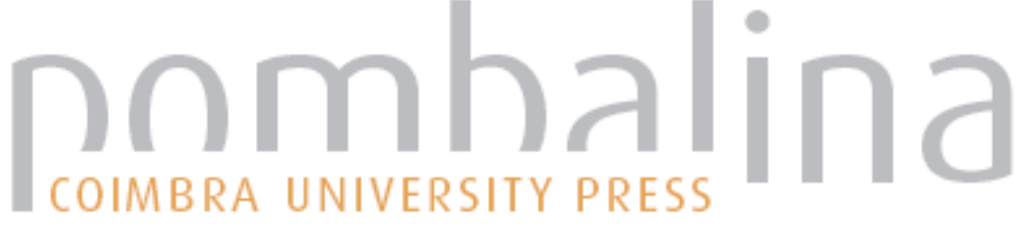

\section{Petrons Kleidernovelle 12-15}

\author{
Autor(es): Lefèvre, Eckard \\ Publicado por: Centro de Estudos Clássicos e Humanísticos \\ URL \\ persistente: URI:http://hdl.handle.net/10316.2/39253 \\ DOI: $\quad$ DOI:http://dx.doi.org/10.14195/978-989-26-1229-4_11
}

Accessed : $\quad$ 26-Apr-2023 13:03:40

A navegação consulta e descarregamento dos títulos inseridos nas Bibliotecas Digitais UC Digitalis, UC Pombalina e UC Impactum, pressupõem a aceitação plena e sem reservas dos Termos e Condições de Uso destas Bibliotecas Digitais, disponíveis em https://digitalis.uc.pt/pt-pt/termos.

Conforme exposto nos referidos Termos e Condições de Uso, o descarregamento de títulos de acesso restrito requer uma licença válida de autorização devendo o utilizador aceder ao(s) documento(s) a partir de um endereço de IP da instituição detentora da supramencionada licença.

Ao utilizador é apenas permitido o descarregamento para uso pessoal, pelo que o emprego do(s) título(s) descarregado(s) para outro fim, designadamente comercial, carece de autorização do respetivo autor ou editor da obra.

Na medida em que todas as obras da UC Digitalis se encontram protegidas pelo Código do Direito de Autor e Direitos Conexos e demais legislação aplicável, toda a cópia, parcial ou total, deste documento, nos casos em que é legalmente admitida, deverá conter ou fazer-se acompanhar por este aviso.

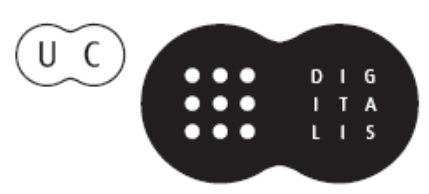


FRANCISCO DE OLIVEIRA

PAOLO FEDELI

DELFIM LEÃO

Coordenadores

\section{- ROMANCE ANTIGO ORIGENS DE UM GÉNERO LITERÁRIO}

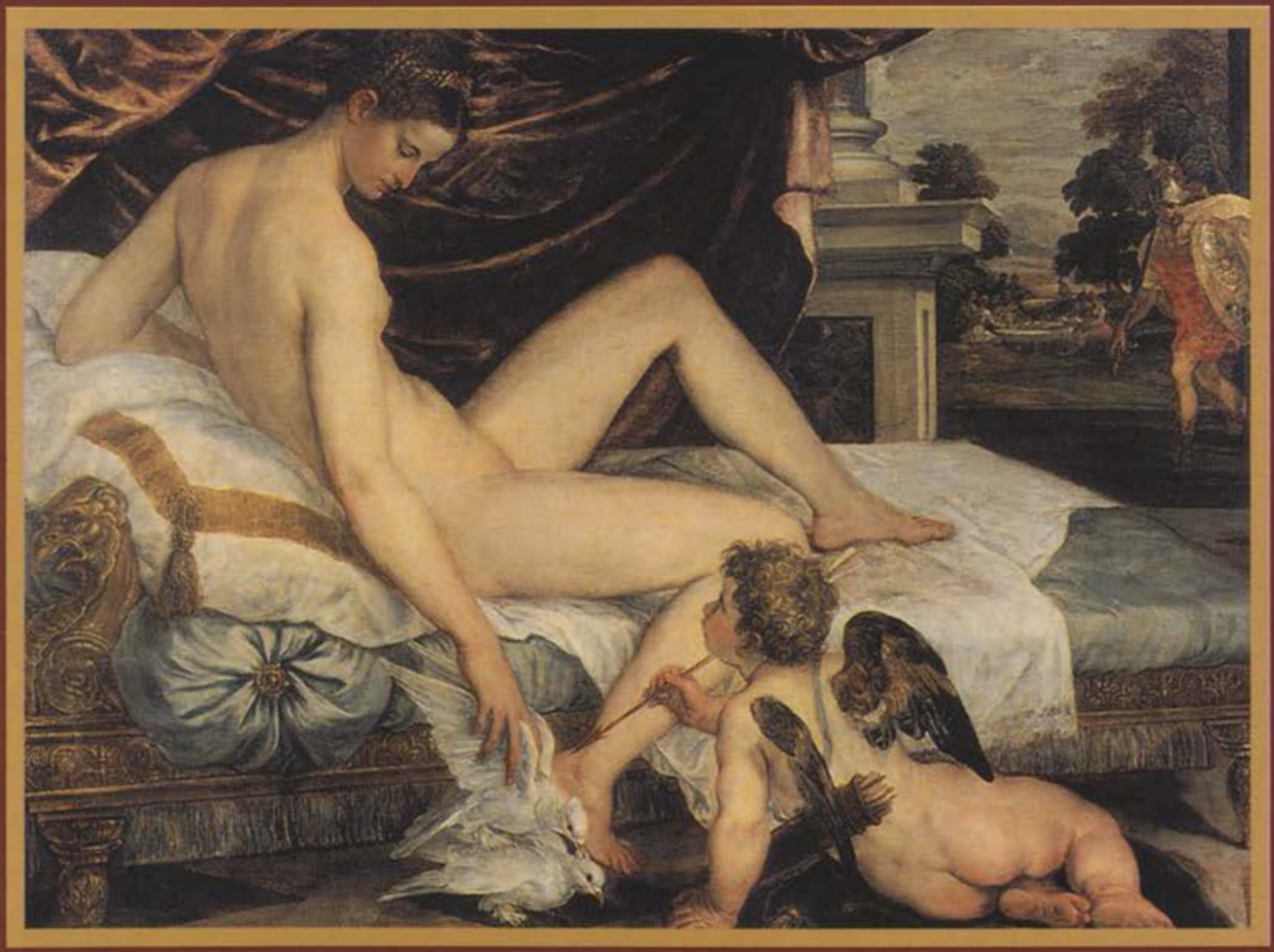

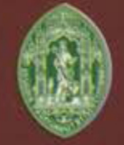

Universidade de Coimbra

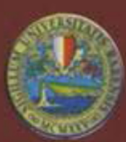

Università degli Studi di Bari

COIMBRA

2005 


\title{
PETRONS KLEIDERNOVELLE 12-15
}

ECKARD LEFĖVRE

Universidade de Freigurg i. B.

\begin{abstract}
Résumé: Wie viele Novellen und Erzählungen Petrons hat auch die Kleidernovelle (Kap. 12-15) einen zweiteiligen Aufbau. Der erste Teil (Kap. 12-15a) hat einen ruhigen ursprünglichen Erzählduktus, während der zweite Teil (Kap. 15, 2b - 15, 9) in lebhafter, ja komplizierter Weise eine Steigerung des ersten Teils bedeutet. Da der erste Teil für sich selbst verständlich ist, könnte es sich um eine bekannte Geschichte handeln, während der zweite Teil von Petron stammt. Es ist möglich, daß der erste Teil auf einen Mimus zurückgeht. Der zweite Teil scheint zeitgenössische juristische Verhältnisse zu parodieren.
\end{abstract}

Die Satyrica reihen Episode um Episode wie Perlen an einer Kette auf. Zum einen gestaltet Petron überlieferte - meist wohl "milesische" - Novellen neu und gibt ihnen durch die Anfügung eines überraschenden Schlusses jeweils einen persönlichen Duktus.' Zum anderen trägt er erfindungsreich, wie zu vermuten ist, eigene Erzählungen vor, die er nach demselben Prinzip wie die überkommenen Novellen komponiert, indem er mit einer pointierten Fortsetzung den jeweils ersten Erzählabschnitt steigert. ${ }^{2}$

$\mathrm{Zu}$ der zweiten Gruppe scheint die Kleidernovelle 12-15 zu gehören. Sie ist, soweit sie vorliegt, von dem Excerptor L durchgängig wiedergegeben. Nur am Ende von 15, 5 und von 15, 7 nehmen Bücheler bzw. Pithou kleinere Lücken an. Es dürfte sich um wenige Wörter handeln.

Die Vorgeschichte ist bei den beiden Streitgegenständen, um die es geht, nicht überliefert. Es verwundert nicht, daß Nodot sie phantasievoll auffüllt. Folgendes ist aus der erhaltenen Erzählung

\footnotetext{
${ }^{1}$ Vgl. LEFÈVRE (1997).

${ }^{2}$ Vgl. LefÉVRe (1997) 34-42 (Petron 126-139); (2003) 147-157 (Petron 61, $8-64,1)$.
} 
heraus zu rekonstruieren. Encolpius und Ascyltos tragen einen geraubten Mantel (raptum latrocinio pallium, 12, 2) in der Dämmerstunde (deficiente iam die, 12, 1) auf einen Trödelmarkt, um ihn zu verkaufen. Der Diebstahl könnte vorher geschildert worden sein; er ist aber auch ohne weitere Erklärung verständlich. Doch dann heißt es von dem hinzutretenden Bauern, er komme Encolpius bekannt vor, er sei quidam familiaris $(12,3)$. Gleich darauf sagt Encolpius noch deutlicher: videbatur ille mihi esse qui tuniculam in solitudine invenerat. plane is ipse erat $(12,5)$. Zwar wäre es möglich, daß er die tunicula sähe und darauf schlösse, es müsse sich um den Finder des Kleidungsstücks handeln, aber das ist nicht wahrscheinlich. Offenbar erkennt er den Bauern wieder. Trifft diese Vermutung zu, ist die Vorgeschichte nicht erhalten.

Ebenso unsicher ist es, ob der S c h 1 u $\beta$ überliefert ist:

1. Die Wendung recuperato, ut putabamus, thesauro $(15,8)$ klingt so, als ob noch eine Überraschung käme, bei der sich herausstellt, daß die Helden doch nicht ihren Schatz wiedererlangt haben. $^{3}$ Jedoch kann sich die Bemerkung auf einen späteren Handlungsabschnitt beziehen, in dem das Gold durch irgendein anderes Ereignis abhanden kommt, wovon aber die Struktur der geschlossenen Novelle nicht berührt wird.

2. Der Bauer wirft zwar Ascyltos die Tunica in das Gesicht, läßt aber Encolpius und Ascyltos den Mantel deponieren (deponere, 15, 7). Der denkende Rezipient erwartet folgerichtig eine Fortsetzung. Es könnte zum Beispiel sein, daß die beiden Gauner als Diebe überführt werden und eine Strafe bezahlen müssen, die etwa dem in der Tunica enthaltenen Gold entspricht. Jedoch braucht nicht mehr gemeint zu sein, als daß der Erzähler ganz allgemein den Standpunkt vertritt, das Geld gehe doch wieder verloren ("wie gewonnen, so zerronnen"). Auch in diesem Fall wäre die Novelle durchaus etwas Abgeschlossenes.

3. Ein vertracktes Problem ist es, ob die mulier aperto capite $(14,5)$ aus der Novelle mit der mulier operto capite $(16,3)$ aus dem Beginn der folgenden Quartilla-Episode identisch ist, was der Überlieferung entspricht und von der Forschung öfter behauptet wird. ${ }^{4}$ Das ist aber wenig wahrscheinlich. Der Bauer betritt den Schauplatz mit einer muliercula $(12,3)$, die bald darauf ihre Kopfbedeckung lockert, um den Mantel genauer mustern zu können $(14,5)$. Wenn es

\footnotetext{
Vgl. Aragosti (1979) 114 Anm. 30.

4 VAN Thiel (1971) 29 Anm. 4; Pellegrino (1986) 195-197.
} 
später heißt, die Wendung der Dinge gefalle dem Bauern und der Frau (rustico mulierique placebat permutatio, 15, 2), dürfte die mulier nichts weiter als seine (moglies sein. ${ }^{5}$ Die Kleidernovelle ragt also nicht in die Quartilla-Erzählung hinüber.

4. Die beiden Hendekasyllabi in 15, 9 bilden wohl kein ganzes Gedicht. «The rest of the poem has been cut out by the excerptor», stellt COURTNEY lapidar fest. ${ }^{6}$ Jedoch werden die restlichen Verse keine neuen Wendung der Handlung gebracht haben.

Ungeachtet des Fehlens der Vorgeschichte und des Schlusses ist die abenteuerliche Geschichte voll aus sich heraus verständlich.

Der Witz der Novelle beruht auf ihrem parallelen A ufbau. Zwei Handlungsstränge entwickeln sich zunächst selbständig und werden dann durch den - in der Welt des Schelmenromans topischen ${ }^{7}$ - Z u fall zusammengeführt. o lusum fortunae mirabilem! $(12,6) \mathrm{ruft}$ der Erzähler aus, als Ascyltos bei dem Bauern die verlorene Tunica entdeckt. Da sie Encolpius abhanden gekommen war, wird er nunmehr durch den Zufall von dem schändlichen Verdacht einer Unterschlagung gereinigt: fortuna me a turpissima suscipione dimiserat $(13,4)$. Eine Einzelheit verdient Aufmerksamkeit. ut $i$ occasione opportunissima coepimus heißt es am Anfang $(12,2)$. In der Neuen Komödie unterstützt Tyche denjenigen, der sich bemüht und die Gelegenheit nutzt, nicht den, der die Hände in den Schoß legt. In diesem Sinn geht Ascyltos mit Bedacht zu Werk, ne quid temere faceret $(12,6)$.

Der Zufall bestimmt auch das glückliche Ende, denn beide Parteien haben den Wunsch nach einer Einigung: itaque utriusque partis votum casus adiuvit $(15,6)$. Damit ist der Vorgang gemeint, daß der Bauer auf die wertlose Tunica verzichtet und nur noch der wertvolle Mantel Gegenstand des Streits ist. Er wird bis ,morgen deponiert, was in der Welt der Novelle heißt, die Auseinandersetzung wird auf den Sankt Nimmerleinstag verschoben, da die Helden ein offizielles Verfahren scheuen. Es wird ja angedeutet, daß es sich bei den Depositaren um Betrüger handelt, die mit dem Mantel verschwinden $(15,5) . \mathrm{Da}$ der Bauer dabei zu Schaden kommt, interessiert in natürlicher Weise die Vagabunden nicht.

Die Struktur der Novelle ermangelt der Evidenz. Die Helden haben einerseits eine wertlose Tunica verloren (pannuciam ne centonibus quidem bonis dignam, 14, 7), in die aber Gold eingenäht ist.

\footnotetext{
5 Vgl. MERKELBACH (1963) 191.

COURTNEY (1991) 18.

7 Vgl. Paratore (1933) 41-42; LeÃo (1998) 119-131.
} 
Andererseits haben sie ein wertvolles Pallium gestohlen (pretiosissimam vestem, 14, 7). Als sie den Mantel auf einem Trödelmarkt verkaufen wollen, entdeckt die Frau eines potentiellen Käufers, daß es sich um ein ihnen gestohlenes Kleidungsstück handelt. Es kommt zu einem heftigen Streit, der andauert, bis Ascyltos zur Güte einen weisen Vorschlag macht: «videmus» inquit "suam cuique rem esse carissimam; reddant nobis tunicam nostram et pallium suum recipiant.» [...] rustico mulierique placebat permutatio $(15,1-2)$. An dieser Stelle könnte - für modernes Empfinden: sollte - die Novelle schließen. Der Bauer muß froh sein, das kostbare Kleidungsstück ohne Rechtsstreit wiederzuerlangen, und die Helden müssen froh sein, nicht wegen Diebstahls verurteilt zu werden und doch ihr Gold wiederzubekommen. Sie nutzen optimal den Zufall, der sich ihnen bietet. Die Geschichte geht sogar "gerecht" aus: Des gestohlenen Mantels gehen die Schelme wieder verlustig, und sie erlangen ihr verlorenes Gut zurück. Daß sie für den Diebstahl nicht bestraft werden, ist für sie ein Vorteil. Das ist insgesamt eine "runde" Geschichte.

Doch dann wird die Auseinandersetzung kompliziert und verliert an Zielgerichtetheit. Die cociones, die vorher nur beiläufig erwähnt wurden $(14,7)$, greifen in das Geschehen ein. cociones sind "Makler". 8 Sie wittern schon in 14, $7 \mathrm{ihr}$ Geschäft. erscheinen advocati nocturni $(15,12)$. Es ist umstritten, ob advocati Partizip oder Substantiv ist. Im ersten Fall werden nocturni herbeigerufen, vielleicht die 'Tresviri capitales" (die «herbeigerufene Streife» ${ }^{10}$ ). Im zweiten - wahrscheinlicheren - Fall erscheinen Advokaten, die als Nachtgesindel bezeichnet werden (nocturni); die letzte Qualifizierung wird durch das überlieferte iam paene etwas eingeschränkt: «des gens de loi, ou plutôt des voleurs de nuit». ${ }^{11}$ Die weitere Charakterisierung qui volebant pallium lucri facere paßt besser zu, 'Winkeladvokaten' als zu offiziellen Tresviri capitales. Die advocati nocturni fordern, daß die Streitobjekte bei ihnen niedergelegt werden und ein Richter am nächsten Tag entscheiden solle (flagitabant uti apud se utraque deponerentur ac postero die iudex querellam inspiceret, 15,2). Darauf einigt man sich (iam sequestri placebant, 15, 4). Auch die dazugekommenen Höker gehören offensichtlich zu der zweifelhaften juristischen Halbwelt. Jedenfalls heißt es über einen von ihnen: solebat aliquando etiam causas agere $(15,4)$. Dieser nimmt den Vorschlag der advocati

8 Vgl. Heraeus (1899/1937) 56-57; Aragosti (1979) 109 Anm. 25; Pellegrino (1986) 191.

Vgl. v. DOMASZEWSKI (1892) 160 (Verweis auf MOMMSEN (1887) 594).

EHLERS (1995) 29.

ERNOUt (1950). Vgl. Aragosti (1979) 110. 
teilweise auf, indem er den Mantel ergreift und erklärt, er werde ihn bis morgen aufheben. Dann wird deutlich gesagt, da $\beta$ es offenbar wird, da $\beta$ die Banditen es auf das kostbare Gewand abgesehen haben: ceterum apparebat nihil aliud quaeri nisi ut semel deposita vestis inter praedones strangularetur $(15,5)$.

Das ist ein Spiel mit dunklen Gestalten, deren Identität und Funktion nicht genau zu sondern sind. Möglicherweise ist das auch gar nicht beabsichtigt. Sie sind allesamt praedones - nicht besser als die Helden des Romans. Nimmt man die verwirrende Handlung hinzu, kann man sich des Eindrucks nicht erwehren, hier werde nicht detailliert klimaktisiert, sondern global kumuliert.

In ähnlicher Weise wird eine Zweiteiligkeit der Novelle von CIAFFI herausgestellt, nach dem die (prima parte) allerdings schon mit 14, 7 schließt: «Ciò che segue, proposta pacificatrice di Ascilto prima, intervento dei cociones poi, soluzione infine all'apparenza favorevole per i nostri eroi, è uno sviluppo mimicamente assai vivo, ma già secondario della scena, che dal punto di vista comico si scarica tutta nella scintilla che scoppia tra le due coppie [sc. Encolpio / Ascilto e rusticus / muliercula] e le rivela nel gioco dell'inversione una identica all'altra» (Sperrung ad hoc). ${ }^{12}$ Die wichtige Beobachtung zur Struktur der Novelle wird quellenanalytisch nicht ausgewertet.

Petron dürfte mit dem "angehängten" Schluß zwei Absichten verfolgen. Die erste kann aus dem ersten Gedicht, den drei Distichen 14, 2, erschlossen werden:

quid faciunt leges, ubi sola pecunia regnat aut ubi paupertas vincere nulla potest?

ipsi qui Cynica traducunt tempora pera nonnumquam nummis vendere verba solent.

5 ergo iudicium nihil est nisi publica merces, atque eques in causa qui sedet empta probat.

Was gelten die Gesetze, wo allein das Geld herrscht oder wo keine Bescheidenheit Oberhand gewinnen kann?

Selbst die Philosophen mit dem kynischen Ranzen, die die Zeit kritisieren, pflegen zuweilen ihre Worte zu verkaufen.

$5 \quad$ Also ist das Gerichtsurteil nichts als eine feile Ware, und der Ritter, der dem Prozeß vorsitzt, billigt gekaufte Stimmen.

Das ist eine Satire auf die gegenwärtige Justiz. Sie ist zeitlos, und es ist konsequent, daß sie auch den Beifall des Excerptor O findet. In derselben Weise wie das Gedicht ist das Tohuwabohu, das durch die

12 CiAfFi (1955) 31. 
advocati nocturni und den cocio, der zuweilen Prozesse führt, entsteht, eine Satire auf die Justiz. Sind in 14, 2 die "hohen" Organe im Visier, geht es in 15, 2-8 um die 'niederen' Chargen. Leider ist der Text nicht immer klar, und zudem sind die einzelnen Gruppen nicht eindeutig zu bestimmen. Der Abschnitt gewönne mit Sicherheit auch für den modernen Leser an Pointiertheit, wenn er die Anspielungen wie Petrons Hörer und Leser auflösen könnte. Einiges ist aber noch erkennbar. In Senecas Apocolocyntosis 12, 2 werden in der Schilderung von Claudius' funus die zwielichtigen causidici den wahren iurisconsulti gegenübergestellt: Agatho et pauci causidici plorabant, sed plane ex animo. iurisconsulti e tenebris procedebant, pallidi, graciles, vix animam habentes, tamquam qui tum maxime revivescerent. ex his unus, cum vidisset capita conferentes et fortunas suas deplorantes causidicos, accedit et ait: 'dicebam vobis: non semper Saturnalia erunt.' Die "Sachwalter", die den petronischen advocati / cociones entsprechen, erscheinen in denkbar negativem Licht. In diesem Sinn sagt Tacitus über die advocati unter Claudius: nec quicquam publicae mercis tam venale fuit quam advocatorum perfidia (Ann. 11, 5, 2). Zwar gelobt Nero bei seinem Amtsantritt Besserung, ${ }^{13}$ aber die juristischen Verhältnisse werden sich nicht von heute auf morgen gebessert haben. Auch ist unklar, wann die einzelnen Partien der Satyrica entstehen. Insofern darf man auch in dieser Passage zeitbezogene Satire vermuten.

Petron liegt aber eine mit dem erhobenen Zeigefinger geäußerte Kritik eines Moralisten fern; «egli [sc. Ascyltos; wir dürfen sagen: Petron] assume la maschera del moralista per rendere più saporosa e arguta la sua trovata. Esula da questi distichi ogni intenzione sia pur larvata o indiretta di propaganda morale. Non era del temperamento di P. fare il satirico dei costumi, essere l'autore di un Sittenroman.») ${ }^{14}$ Petron geht es um das ridentem dicere verum, wobei auf dem ersten Wort ein besonderes Gewicht liegt. Petron steht Horaz näher als Seneca.

Die zweite Absicht, die Petron mit dem "angehängten" Schluß verfolgt, scheint aus dem zweiten Gedicht, den beiden Hendekasyllabi 15,9 , hervorzugehen:

nolo quod cupio statim tenere,

nec victoria mi placet parata.

Was ich wünsche, will ich nicht sofort erringen, nicht gefällt mir ein leichter Sieg.

13 Vgl. Tac. Ann. 13, 5, 1.

14 PARATORE (1933) 42. 
Was dem modernen Leser bei der ausgesponnenen Erzählung etwas nojos erscheint, ist für Petron, jedenfalls für seinen Helden Ascyltos, der Clou. Dieser ist der Verhandlungsführer und zieht wohl auch das Fazit. ${ }^{15}$ Es lautet: «Ich wünsche mir keinen zu schnellen Erfolg, mir gefallen Schwierigkeiten; sie sind dazu da, überwunden zu werden.» Wenn diese Einstellung Petrons Konzeption seiner Helden widerspiegelt, muß ihm der einfahe Tausch der Kleidungsstücke in 15, $2 \mathrm{zu}$ matt - als eine victoria parata - erscheinen. Und doch ist der "erste Schluß", der mit Hilfe des Logos herbeigeführt wird, eleganter als die abermalige Bemühung des Zufalls (casus adiuvit, 15, 6) in dem "zweiten Schluß", bei dem natürlich die alte römische Maxime fortem fortuna adiuvat ${ }^{16}$ mitzuhören ist.

Ob es sich bei der Novelle 12-15, 2a um eine bekannte Erzählung handelt, die Petron im einzelnen ausgestaltet - zum Beispiel durch das Gedicht 14, 2 - und mit dem nach seinem Empfinden steigernden "zweiten Schluß" $(15,2 b-15,9)$ ausstaffiert, muß offen bleiben. Sofern die Milesischen Novellen nur erotischen Inhalts waren, ${ }^{17}$ wäre das "Original" anderswo zu suchen. Naheliegend ist die Herleitung aus dem Mimus: ${ }^{18}$ "se altre scene del romanzo mostrano maggiore vis farsesca, questa, per la scelta dell'argomento, per i personaggi che vi sono introdotti, è forse quella che più è ispirata, per non dire imitata, dal mimo, e sopratutto dal mimo rustico, dalla farsa campagnuola. Ciò che nella rappresentazione mimica era affidato al gesto, al giuoco scenico, qui P., con sforzo mirabile, ha tentato di sostituire con la parola, che in questo luogo è nient'altro che didascalia (vigorosa e plastica didascalia) alle poche parole in discorso diretto. E difatti la scena non è descritta come si usa fare di solito nella prosa novellistica: ciò che vien colto sono $\mathrm{i}$ varii atteggiamenti mimici dei personaggi, quegli atteggiamenti appunto su cui si basava l'azione nella farsa recitata. Sembra addirittura che P. abbia rivissuto nella fantasia un'azione scenica, in tutte le sue movenze, in tutti i suoi gesti, e abbia fedelmente trascritto in forma narrativa proprio questo complesso mimico sgorgato dalla sua stessa sbrigliatissima vena comica.» ${ }^{19}$ Wenn diese Charakterisierung auf Petrons Schaffensprozeß zutrifft, muß man aus ihr folgern, daß nur der klare erste Teil einen Mimus wiedergeben

15 Paratore (1933) 47; Pellegrino (1986) 195.

16 Vgl. Terenz Ph. 203 (fortes fortuna adiuvat).

17 Vgl. LeFĖVRE (1997) 5-6.

18 Vgl. Aragosti (1979) 104 Anm. 10.

19 Paratore (1933) 40-41. 
kann, nicht aber der unklare zweite Teil, dessen verschiedene Personengruppen nicht in evidenter Weise szenisch ("mimisch") darstellbar sind. Denn wie sollte man advocati nocturni, sequestri und cociones so vorführen, daß die Zuschauer wissen, um wen es sich handelt? Der angehängte Schluß 15, 2b-15, 9 ist ein typisches Erzeugnis narrativer Schriftlichkeit.

Andererseits spricht nichts dagegen, daß Petron auch eigene Erfindungen wie überkommene in der dargelegten Weise komponiert.

\section{Literaturverzeichnis}

ARAGOSTI, A., "L'episodio petroniano del forum (Sat. 12-15): assimilazione dei codici nel racconto", MD 3.(1979) 101-109.

BüCheler, F. (ed.), Petronii Saturae (Berolini 1862, $\left.{ }^{4} 1904\right)$.

ClAfFI, V., Struttura del Satyricon (Università di Torino, Pubblicazioni della Facoltà di lettere e filosofia 7, 1, Torino 1955).

Courtney, E., The Poems of Petronius (American Classical Studies 25, Atlanta 1991).

DOMASZEWSKI, A. v., "Nocturni", RhM 47 (1892) 159-160.

Ehlers, W. / Müller, K. (ed.), Petronius, Satyrica, Schelmenszenen (Lateinisch deutsch, zugleich Zürich und Darmstadt 1995).

Ernout, A. (ed.), Pétrone (Paris $\left.{ }^{3} 1950\right)$.

Heraeus, W., "Die Sprache des Petronius und die Glossen", (Progr. Offenbach a. M. 1899), berichtigt und vermehrt in: DERS., Kleine Schriften (Heidelberg 1937, 52$150)$.

LEÃo, D. Ferreira, As Ironias da Fortuna. Sátira e Moralidade no Satyricon de Petrónio, Lisboa 1998.

LefĖvre, E., Studien zur Struktur der ,Milesischen' Novelle bei Petron und Apuleius (AAWM, Geistes- und Sozialwiss. Kl. 5, 1997, Stuttgart 1997).

LefÈvre, E., "Petrons Spuknovellen 61, 8-64, 1", in Petroniana. Gedenkschrift für H. PETERSMANN, ed. J. Herman / H. Rosén (Heidelberg 2003, 147-157).

MerkelbaCH, R., "Eine Lücke im Petrontext (Kap. 15)", RhM 106 (1963) 191-192.

Mommsen, TH., Römisches Staatsrecht, 11 / 1 (Leipzig ${ }^{3} 1887$ ).

Müller, K. (ed.), Petronius, Satyricon Reliquiae. (Editio iterata correctior editionis quartae (MCMXCV), Monachii et Lipsiae 2003).

Paratore, E., Il Satyricon di Petronio, (II: Commento, Firenze 1933).

Pellegrino, C. (ed.), T. Petronio Arbitro, Satyricon. Introduzione, testo critico, commento (I: I capitoli della retorica, Roma 1986).

VAN Thiel, H., Petron. Überlieferung und Rekonstruktion (Mnemosyne Suppl. 20, Lugduni Batavorum 1971). 